\title{
EIT AND LASCO OBSERVATIONS OF THE INITIATION OF A CORONAL MASS EJECTION
}

\author{
K. P. DERE ${ }^{1}$, G. E. BRUECKNER ${ }^{1}$, R. A. HOWARD ${ }^{1}$, M. J. KOOMEN ${ }^{1, a}$, \\ C. M. KORENDYKE ${ }^{1}$, R. W. KREPLIN ${ }^{1, b}$, D. J. MICHELS ${ }^{1}$, J. D. MOSES ${ }^{1}$, \\ N. E. MOULTON ${ }^{1, c}$, D. G. SOCKER ${ }^{1}$, O. C. ST. CYR ${ }^{1, d}$, J. P. DELABOUDINIÈRE ${ }^{2}$, \\ G. E. ARTZNER ${ }^{2}$, J. BRUNAUD ${ }^{2}$, A. H. GABRIEL ${ }^{2}$, J. F. HOCHEDEZ ${ }^{2}$, \\ F. MILLIER ${ }^{2}$, X. Y. SONG ${ }^{2}$, J. P. CHAUVINEAU ${ }^{3}$, J. P. MARIOGE ${ }^{3}$, J. M. DEFISE ${ }^{4}$, \\ C. JAMAR ${ }^{4}$, P. ROCHUS ${ }^{4}$, R. C. CATURA ${ }^{5}$, J. R. LEMEN ${ }^{5}$, J. B. GURMAN ${ }^{6}$, \\ W. NEUPERT ${ }^{6}$, F. CLETTE ${ }^{7}$, P. CUGNON ${ }^{7}$, E. L. VAN DESSEL ${ }^{7}$, P. L. LAMY ${ }^{8}$,

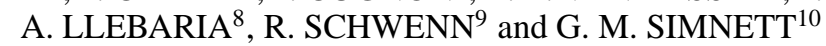 \\ ${ }^{1}$ E.O. Hulburt Center for Space Research, Naval Research Laboratory \\ Washington D.C., 20375-5320 U.S.A. \\ ${ }^{2}$ Institut d'Astrophysique Spatial, Université Paris XI, 91405 Orsay, France \\ ${ }^{3}$ Institut d'Optique Théorique et Appliquée, 91403 Orsay, France \\ ${ }^{4}$ Centre Spatial de Liège, Liège, Belgium \\ ${ }^{5}$ Lockheed Martin Palo Alto Research Laboratory, Palo Alto, CA 94304 U.S.A. \\ ${ }^{6}$ NASA Goddard Space Flight Center, Greenbelt, MD 20771 U.S.A. \\ ${ }^{7}$ Observatoire Royal de Belgique, Brussels, Belgium \\ ${ }^{8}$ Laboratoire d'Astronomie Spatiale, Marseille, France \\ ${ }^{9}$ Max-Planck-Institut für Aeronomie, Lindau, Germany \\ ${ }^{10}$ Space Research Group, School of Physics and Space Research \\ University of Birmingham, Birmingham, U.K.
}

(Received 18 March 1997; accepted 18 June 1997)

\begin{abstract}
We present the first observations of the initiation of a coronal mass ejection (CME) seen on the disk of the Sun. Observations with the EIT experiment on SOHO show that the CME began in a small volume and was initially associated with slow motions of prominence material and a small brightening at one end of the prominence. Shortly afterward, the prominence was accelerated to about $100 \mathrm{~km} \mathrm{~s}^{-1}$ and was preceded by a bright loop-like structure, which surrounded an emission void, that traveled out into the corona at a velocity of $200-400 \mathrm{~km} \mathrm{~s}^{-1}$. These three components, the prominence, the dark void, and the bright loops are typical of CMEs when seen at distance in the corona and here are shown to be present at the earliest stages of the CME. The event was later observed to traverse the LASCO coronagraphs fields of view from 1.1 to $30 R_{\odot}$. Of particular interest is the fact that this large-scale event, spanning as much as $70 \mathrm{deg}$ in latitude, originated in a volume with dimensions of roughly $35^{\prime \prime}\left(2.5 \times 10^{4} \mathrm{~km}\right)$. Further, a disturbance that propagated across the disk and a chain of activity near the limb may also be associated with this event as well as a considerable degree of activity near the west limb.
\end{abstract}

\section{Introduction}

Coronal mass ejections (CMEs) are often seen as spectacular eruptions of matter from the Sun which propagate outward through the heliosphere and often interact

\footnotetext{
${ }^{\text {a }}$ Sachs Freeman Assoc.

${ }^{\mathrm{b}}$ Universities Space Research Assoc.

${ }^{c}$ Allied Signal Corp.

${ }^{\mathrm{d}}$ Computational Physics Inc.
}

Solar Physics 175: 601-612, 1997.

(C) 1997 Kluwer Academic Publishers. Printed in Belgium. 
with the Earth's magnetosphere. The nature and cause of CMEs is a fundamental, unsolved problem in solar physics. Often they are associated with prominence eruptions and/or solar flares. The classical CME consists of 3 parts: a bright coronal loop, a dark void, and the eruptive prominence (Kahler, 1987). Other reviews of CMEs include those by Wagner (1984) and Hundhausen (1987). In the past, essentially all coronal mass ejections have been observed by coronagraphs that occult the emissions from the disk of the Sun in order observe the much weaker photospheric light that has been Thomson-scattered by coronal electrons. Consequently, it has not been possible to observe the earliest stages of a CME, aside from the eruptions of the prominence material which can be observed in $\mathrm{H} \alpha$. We present here the first observations of all 3 components of a coronal mass ejection during its initiation phase.

\section{Observations}

The Solar and Heliospheric Observatory (SOHO), a joint European Space Agency (ESA) and National Aeronautics and Space Administration (NASA) effort, was launched late in 1995 and has provided unprecedented observations of the Sun and heliosphere. In this paper, we present observations made by 2 of the instruments on SOHO, the Extreme Ultraviolet Imaging Telescope (EIT) and the Large Angle Spectrometric Coronagraph (LASCO). A detailed description of these instruments is provided by Delaboudinière et al. (1995) and Brueckner et al. (1995).

Briefly, the EIT consists of a Ritchey-Chrétien telescope with EUV reflecting multilayer coatings, several filters and a CCD detector. Different multilayer coatings are placed on 4 quadrants of both the primary and secondary mirrors in order that any 1 of 4 wavelengths ranges can be observed at a given time, while providing a good registration of images obtained in the four wavelengths. The four wavelengths and dominant emitters are $171 \AA$ (Fe IX and Fe X), $195 \AA$ (Fe XII), $284 \AA$ (Fe XV) and $304 \AA$ (He II). The EIT observations that we will discuss were obtained with the $195 \AA$ Fe XII channel. Fe XII is emitted most efficiently at temperatures near $1.5 \times 10^{6} \mathrm{~K}$. A strong Fe XXIV line at $192 \AA$ could also contribute to the $195 \AA$ channel signal if $1.5 \times 10^{7} \mathrm{~K}$ plasmas, as can occur in solar flares, are in the line of sight. Another contributor to the observed pattern of emission at $195 \AA$ is the presence of absorbing features. Absorption of coronal emissions is often noticed in all 3 of the coronal channels at locations of prominences and macrospicules, both seen in He II. The mechanism for this absorption is probably photo-ionization of $\mathrm{He}$ I at wavelengths below $504 \AA$ and of He II at wavelengths below $228 \AA$. This turns out to be a particularly useful feature since, in this case, it allows one to see both the prominence and coronal structures in the CME.

The LASCO instrument consists of an internally occulted coronagraph, C1, and 2 externally occulted coronagraphs, $\mathrm{C} 2$ and $\mathrm{C} 3$. The $\mathrm{C} 1$ coronagraph is able to observe the corona to within about $1.1 R_{\odot}$ with a field of view that extends 
to about $3 R_{\odot}$. Observations with $\mathrm{C} 1$ are made through a tunable, narrow-band Fabry-Pérot filter. The passband of the Fabry-Pérot filter is around $0.8 \AA$ wide. The set of observations made with the $\mathrm{C} 1$ coronagraph were made at line-center of the Fe XIV $\lambda 5303$ line and off-band at $5309 \AA$. The field of view of $\mathrm{C} 2$ extends from about $1.5 R_{\odot}$, the edge of the occulter, to $6 R_{\odot}$. Similarly, the $\mathrm{C} 3$ coronagraph has a field of view from 4 to $30 R_{\odot}$. The signal in $\mathrm{C} 2$ and $\mathrm{C} 3$ consists of broad-band visible photospheric light scattered primarily by electrons in the corona.

During the period from 22 December 1996 02:00 UT until 28 December at 16:00 UT, the LASCO and EIT experiments were able to obtain a unique set of observations. For most operations, the combined LASCO and EIT telemetry rate is $5.2 \mathrm{Kbs}^{-1}$. During this period, a large fraction of the SOHO experiment telemetry was dedicated to EIT and LASCO and resulted in a combined telemetry rate of about $25 \mathrm{Kbs}^{-1}$. Consequently, it was possible to obtain and downlink 1 EIT Fe XII image every $12 \mathrm{~min}, 1 \mathrm{C} 1 \mathrm{Fe}$ XIV image every $24 \mathrm{~min}, 1 \mathrm{C} 2$ image every $24 \mathrm{~min}$ and $1 \mathrm{C} 3$ image every $50 \mathrm{~min}$.

\section{The 23 December 1996 Coronal Mass Ejection}

The 23 December 1996 coronal mass ejection originated in NOAA active region 8005. This active region first appeared roughly one solar rotation before the CME studied here. On 24 November 1996, it was first evident as a small brightening in the EIT Fe XII images and early on 25 November it was seen as a small bright region in the EIT He II image. The new magnetic flux emerged into a region of enhanced diffuse flux that was the remnant of a older active region. The orientation of the new flux with respect to the pre-existing flux was such that new field lines connecting the new and old regions could be expected to form. Feynman and Martin (1995) have suggested that these conditions are favorable for the production of CMEs. When viewed earlier near disk center, the Observatory of Paris-Meudon $\mathrm{H} \alpha$ spectroheliograms showed a filament through its center. Solar Geophysical Data indicates the observation of several small sunspots on 16 and 17 December. By the time of the 23 December CME, the region was decaying in EUV intensity and the sunspots were no longer observed. The region did not reappear on the next solar rotation. The joint USAF/NOAA report of solar and geophysical activity indicated a small filament eruption near 14S 67W beginning at 20:16 UT on 23 December. This disappearing filament was located near region 8005 (13S, 67W) and was associated with a small flare (B2/sf) which peaked at 20:53 UT.

The first sign of the CME in the EIT $195 \AA$ images was at 20:20 UT when a small brightening and the motion of a short segment of a prominence were observed. The length of the section of the prominence involved was approximately $35^{\prime \prime}\left(2.5 \times 10^{4} \mathrm{~km}\right)$. The southern end of this segment was displaced from its position at 20:03 UT by about $15^{\prime \prime}\left(1.1 \times 10^{4} \mathrm{~km}\right.$, indicating a velocity of about $11 \mathrm{~km} \mathrm{~s}^{-1}$. The prominence was seen as an absorption feature. Attached to the 


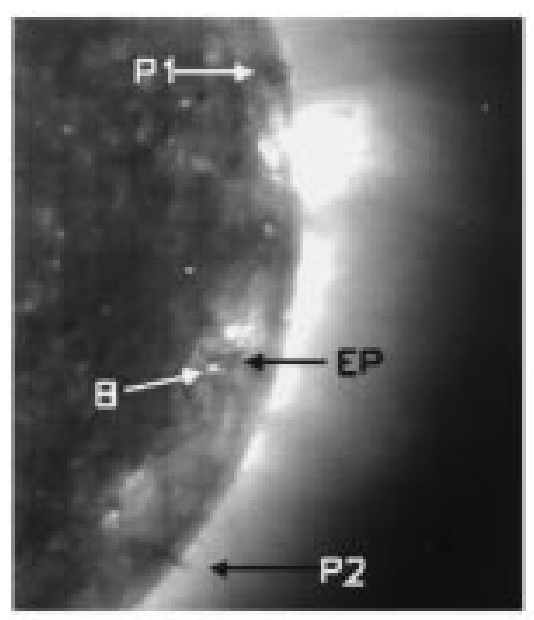

20:20 UT

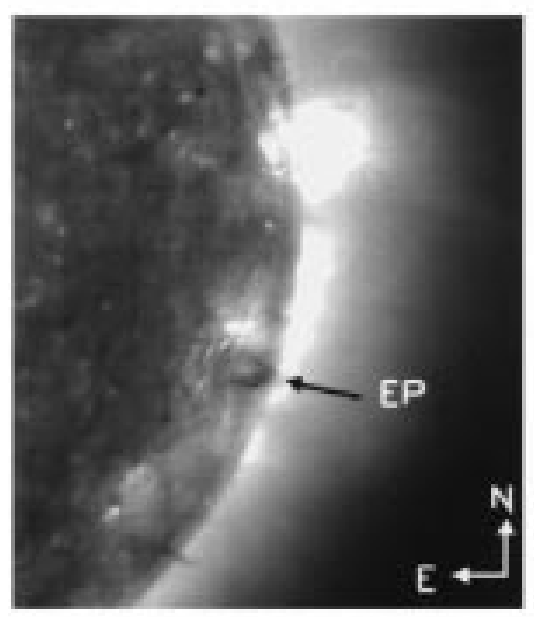

20:41 UT



20:32 UT

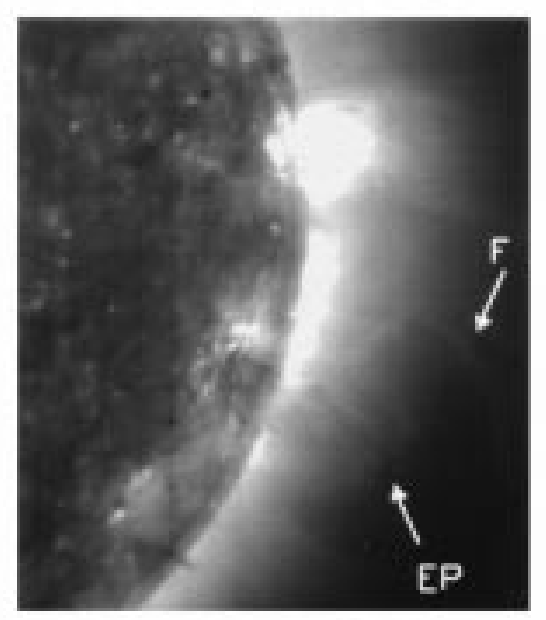

20:53 UT

\section{EIT Fe XII 195^ 23-Dec-1996}

qkl

Figure 1. EIT images of the initiation of a CME. The letters in the figures are used to indicate the following structures: EP, the eruptive prominence; $\mathrm{B}$, the flare-like brightening; P1 and P2, prominences; F, the outer front of the CME. Solar north and east are as indicated in the images at 20:41 UT. 


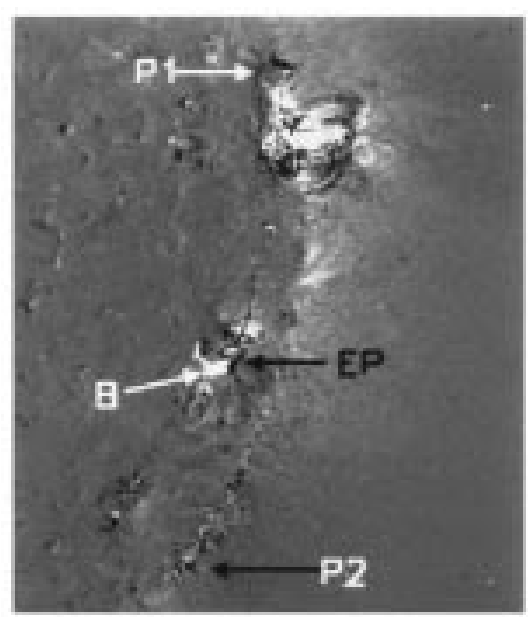

20:20 UT

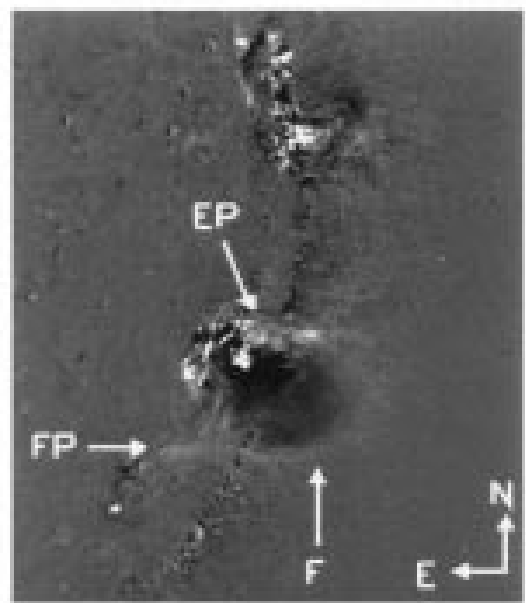

20:41 UT

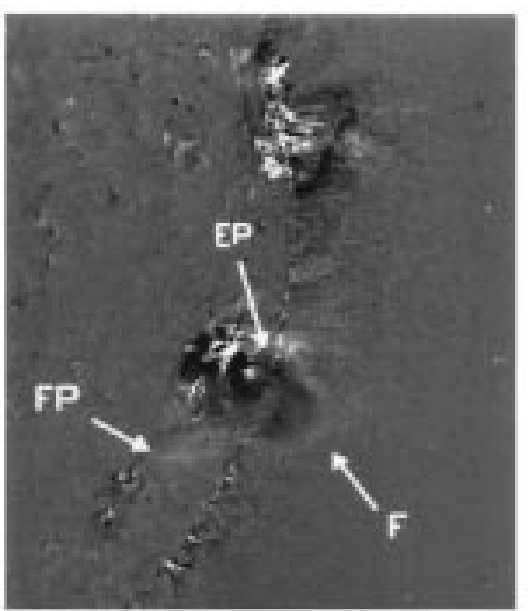

20:32 UT

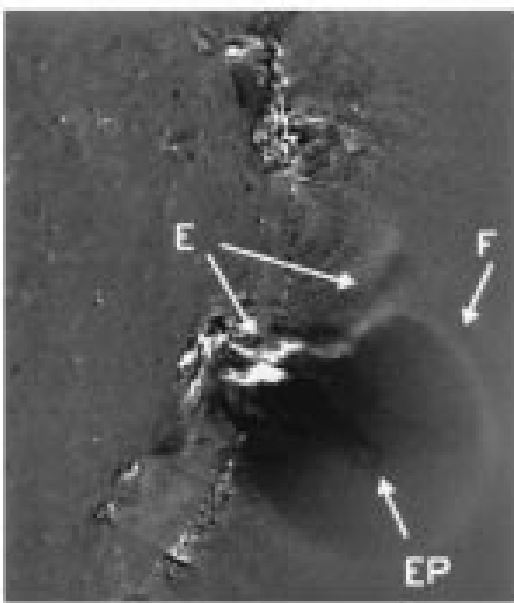

20:53 UT

\section{EIT 195Å 23-Dec-1996}

$q \mathrm{kl}$

Figure 2. EIT running difference images of the initiation of a CME. The letters in the figures are used to indicate the following structures: EP, the eruptive prominence; $\mathrm{B}$, the flare-like brightening; P1 and P2, prominences; F, the outer front of the CME; FP, the suspected footpoint of one side of the CME; E, a secondary set of ejecta. Solar north and east are as indicated in the images at 20:41 UT. 
southern end of the prominence segment is a curved bright linear structure. Near the middle of this bright structure is a much brighter knot of emission. These features can be seen in Figure 1 and Figure 2. Figure 1 shows 4 EIT $195 \AA$ A images at the times indicated. Figure 2 shows running difference images of the EIT $195 \AA$ emission. This bright knot of emission is probably the the site of the X-ray flare observed by the GOES detectors. In each case the image at a given time is the difference from the preceding image, all of which are shown in Figure 1 except for the image at 20:03 UT. Increases in intensity at a given position are indicated by white and decreases by black. Figure 1 best displays the prominence eruption while the difference images in Figure 2 best show the weak coronal emissions associated with the CME.

From the running difference images in Figure 2, as early as 20:32 UT, a weak but relatively bright loop-like structure, surrounding a relatively dim area, can be seen and is evident in the next two frames as it expands outward. Under the assumption that these structures are ejected radially from the Sun, their velocity is on the order of $200 \mathrm{~km} \mathrm{~s}^{-1}$.

The exact geometry of the bright loop-like structure is not clear. This observational problem is related to the question of whether the loop-like structure is a compressional wave that propagates outward through the corona or whether it is the expulsion of coronal field and plasma (Sime and Hundhausen, 1987; Low and Hundhausen, 1987). One clue for distinguishing between the two scenarios would be to examine the footpoints of the apparent loops. The difference images at 20:32 UT and 20:41 UT show a relatively bright stationary elongated structure that appears to be the southern footpoint of the expanding CME loop. This footpoint is denoted by 'FP' in Figure 2. This same location is also consistent with being the location of the footpoint of the loop seen at 20:53 UT. It does not appear possible to identify a northern footpoint of the CME from these images. Just to the north of the prominence seen at 20:20 UT, a set of coronal loops span the neutral line from which the prominence apparently originated. These loops seem to be unaffected by the nearby CME, at least between 20:20 and 20:53 UT. These facts suggest that the bright loop-like structure remains connected to fixed footpoints for a period of time.

As seen in Figure 2, the eruptive prominence starts out as a fairly simple linear structure at 20:20 UT. By 20:32 UT it is considerably more complex. At 20:41 UT it appears as a collection of twisted, kinked filaments. By 20:53 UT it has been extended outward while material continues to be ejected from the base of the CME. In the EIT running difference image at 20:53 UT, bright ejecta, labeled 'E' in Figure 2, can be seen. These would constitute a new phase of material ejection following the initial prominence eruption. This new material is also bright in Fe XII indicating that it is at coronal temperatures as opposed to the cooler temperatures of the prominence material. This new material also seems to be connected to the active region to the north. 


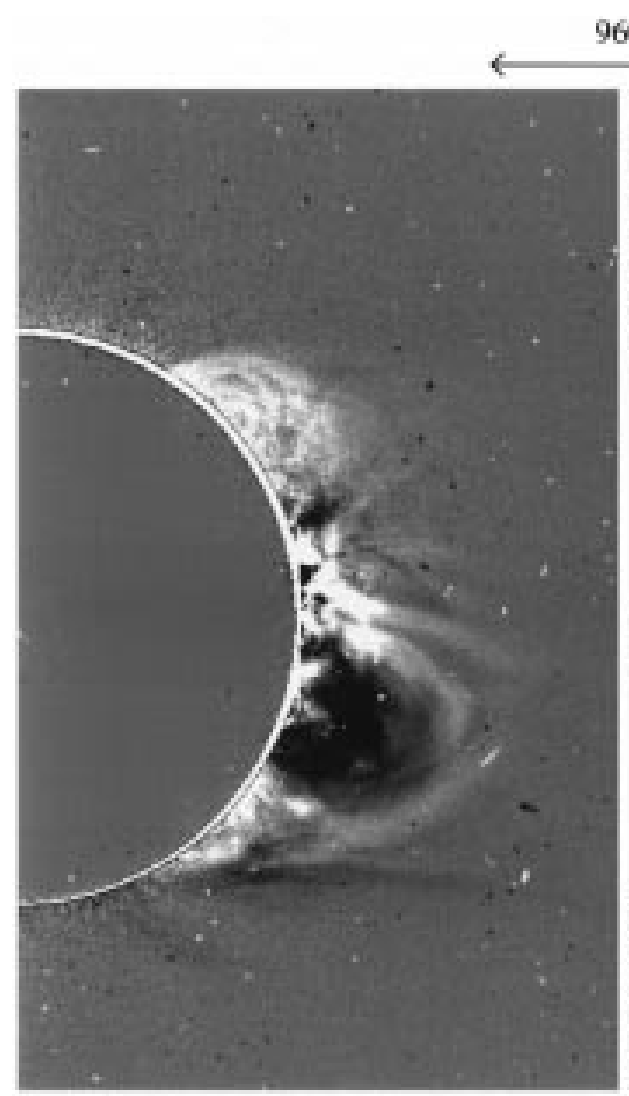

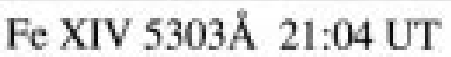

$960^{\circ}$

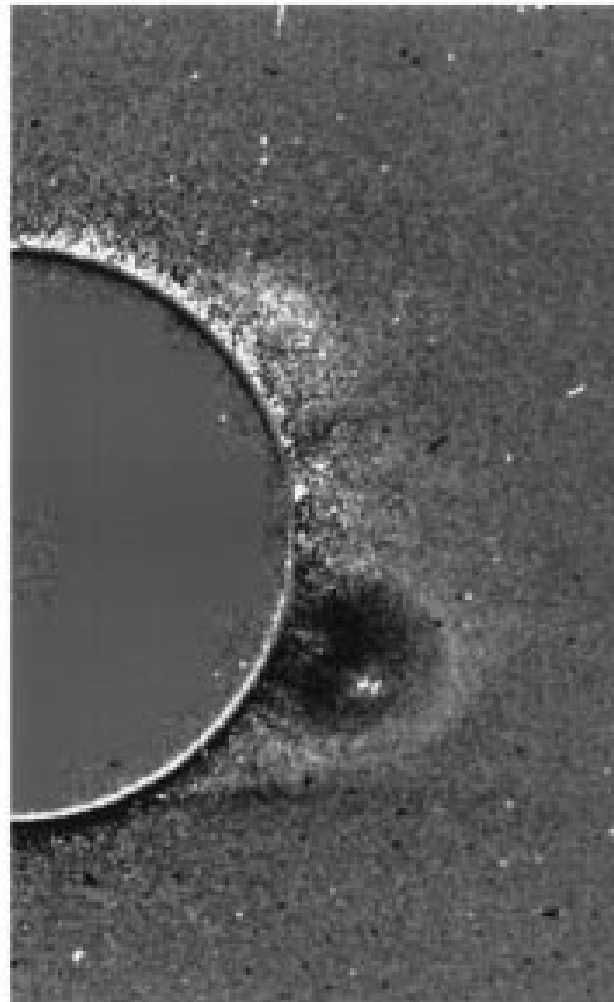

K Corona 5309£ 21:07 UT

\section{LASCO C1 23-Dec-1996}

Figure 3. Images of the CME in the LASCO C1 coronagraph.

There is evidence that a weak large-scale wave passes across the solar disk. Although the signature of this wave is not especially distinct, it appears to originate near the site of the CME and travel outward as a slight brightening. At 21:22 UT the wave is seen to the northeast of the CME site at a distance of $3 \times 10^{5} \mathrm{~km}$, indicating a velocity of $80 \mathrm{~km} \mathrm{~s}^{-1}$, if it is assumed that it was initiated at the same time as the CME.

Beginning at 21:10 UT, the EIT images, not presented here, show the formation of a system of postflare loops, as are often observed following an eruptive prominence or CME (Sheeley et al., 1975; Kahler, 1977; Webb, Krieger, and Rust, 1976; Tsuneta et al., 1992). The pre-event images show a dark linear structure that would appear to mark the neutral line dividing the 2 magnetic polarities of this active region. The post-flare loops do not form along this neutral line but to the east of it. 
As the CME traveled outward, it was observed several minutes later in the $\mathrm{C} 1$ coronagraph, as show in Figure 3. Both panels were obtained by subtracting an earlier image obtained around 18:30 UT from an image at the stated time at the same wavelength. The image on the left was obtained at line center of the Fe XIX $5303 \AA$ coronal emission line. The image on the right was obtained at $5309 \AA$ where the signal is due to scattering of photospheric light by free electrons, the $\mathrm{K}$ corona. Both $\mathrm{C} 1$ images show a distinct bright loop-like structure enclosing a region of decreased intensity. In addition, the K corona image shows an additional bright interior structure that is probably the prominence. The signature of the CME was not seen in the preceding $\mathrm{C} 1$ image at 20:38 UT, nor in the subsequent image at 21:28 UT.

The leading edge of this CME first appears in the C2 field at 21:16 UT, as shown in Figure 4. The images shown in Figure 4 are running difference images wherein the image at the stated time has been differenced from the immediately preceding image. There was a gradual swelling of the existing white-light streamer (particularly on the equatorward side) during the several hours prior to that time. The prominence material first appears in the next $\mathrm{C} 2$ image, taken at 21:37 UT. The angular span of this event as measured in $\mathrm{C} 2$ was $70 \mathrm{deg}$, centered on position angle 250. The images of the CME in $\mathrm{C} 2$ do not appear to have the same loop-like or bubble-like appearance as the images in the EIT or $\mathrm{C} 1$. There is evidence of a bright concave-outward structure appearing late in the mass ejection, and this has been taken to be a signature of disconnecting magnetic field lines by some researchers (Simnett et al., 1997, for example). The original streamer seen in white-light was significantly reduced in size following the CME.

In order to show the development of the CME through the fields of view of the EIT, and $\mathrm{C} 1$ and $\mathrm{C} 2$ coronagraphs, we have traced the outline of the leading edge of the CME as seen in each of the images presented here. This composite is shown in Figure 5 where the circle shows the disk of the Sun. The CME started out with a fairly circular geometry but became more elongated with time when seen later in the $\mathrm{C} 2$ field of view. The height of the CME as a function of time is plotted in Figure 6. The values for the height assume that the CME is moving radially outward from the site where it was first seen on the disk. The velocities in the EIT and $\mathrm{C} 1$ fields of view are on the order of $200 \mathrm{~km} \mathrm{~s}^{-1}$ and $400 \mathrm{~km} \mathrm{~s}^{-1}$ in the $\mathrm{C} 2$ field of view.

\subsection{ACCOMPANYING PHENOMENA}

A number of nearly simultaneous events were observed in the EIT along the SW limb that may or may not be related to the central CME discussed above. While it is not possible to demonstrate any direct connection between these events, they appear to form a sequence. To the north $(82 \mathrm{~W}, 13 \mathrm{~N})$, a prominence (P1 in Figure 1) appears around 19:30 UT on 23 December with increasing size and apparent motion along the disk. A second period of growth of the prominence commences around 


\section{Running difference images}



21:16 UT

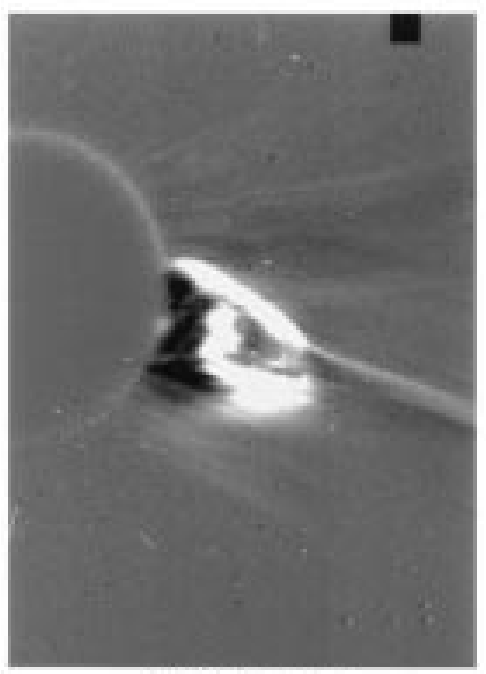

22:06 UT

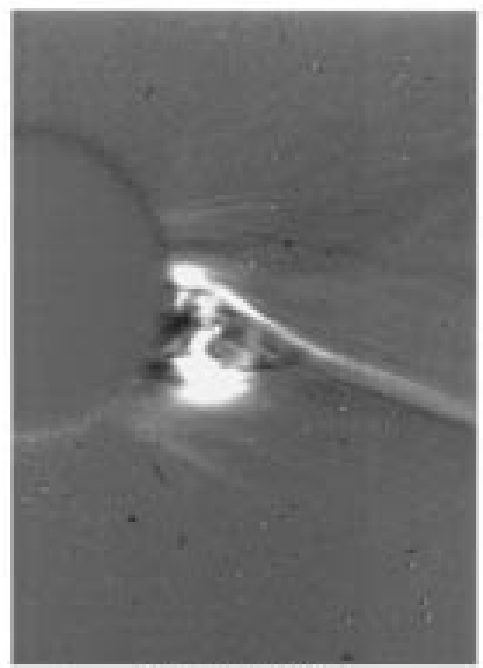

21:37 UT

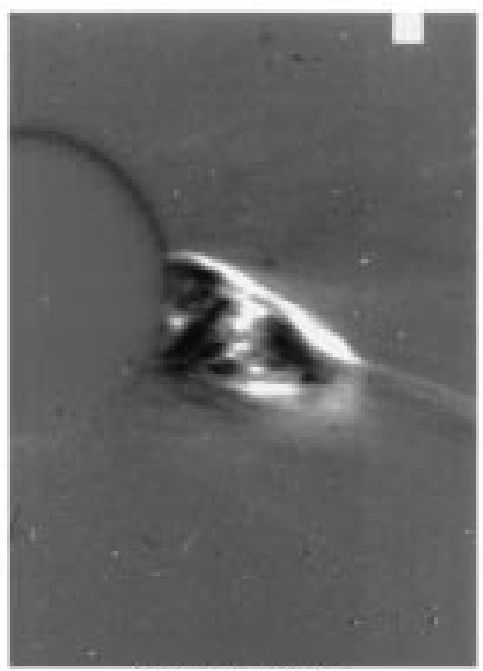

22:27 UT

\section{LASCO-C2 23-Dec-1996}

Figure 4. Images of the CME in the LASCO C2 coronagraph.

00:41 UT on 24 December. An ejection of coronal material appears above this site at around 05:14 UT on 24 December. The prominence and the coronal ejection appear to be associated with NOAA active region 8004 which, when viewed closer to disk 




Figure 5. The leading edge of the 23 December 1996 CME as seen in the EIT, the LASCO C1 and C2 coronagraphs. The numbers can be associated with the time and telescope as follows: 4, 20:53 UT (EIT); 5, 21:04 UT (C1); 6, 2116 (C2); 7, 21:37 UT (C2); 8, 22:06 UT (C2); 9, 22:27 UT (C2); 10, 22:56 UT (C2). The lower lines refer to the front ' $F$ ' seen in the first 4 panels of Figure 2.

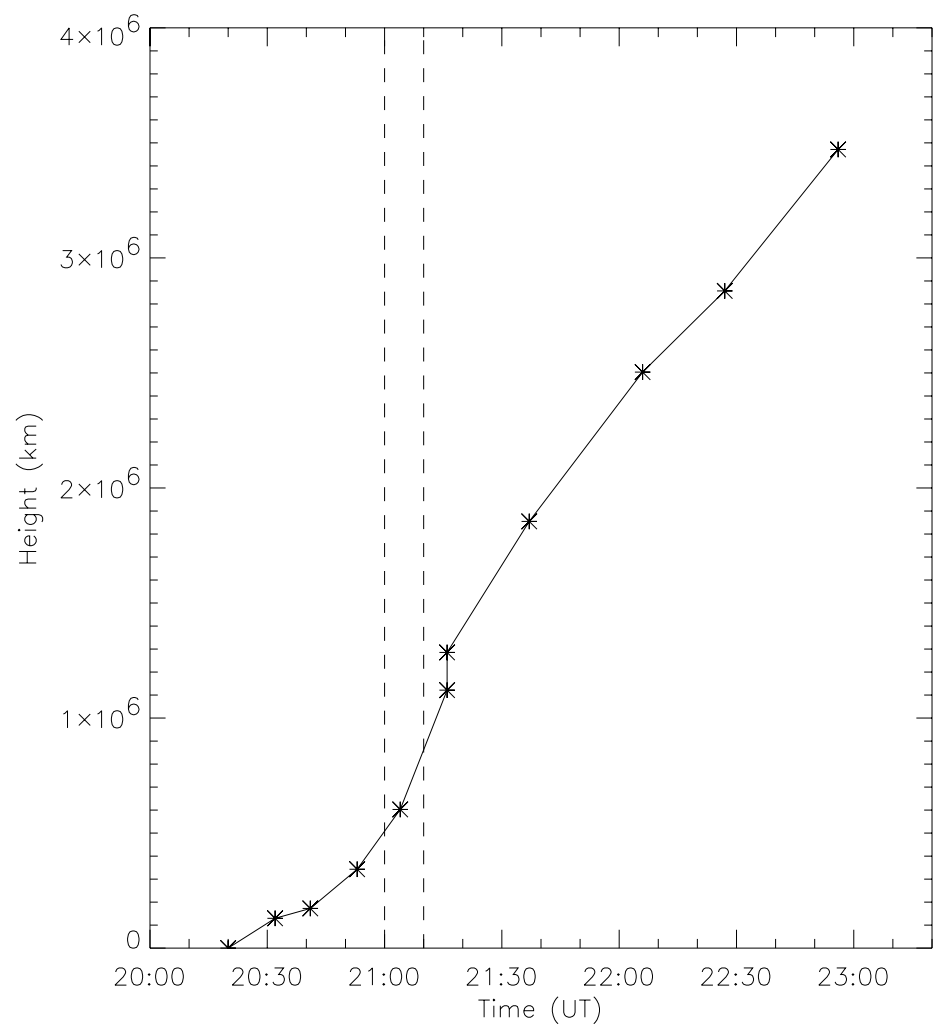

Figure 6. Height of the leading edge as a function of time. The $\mathrm{C} 1$ observations are inside the dashed lines, EIT to the left of it and $\mathrm{C} 2$ to the right.

center, showed an active region prominence through its center. On 21 December, a new active region (NOAA 8007) emerged to the east of NOAA 8004. 
To the south, as seen in Figure 1, an elongated, nearly vertical prominence at $37 \mathrm{~S} 90 \mathrm{~W}$ began to stretch further out into the corona and at 00:31 UT on 24 December the prominence (P2 in Figure 1) was ejected outward into the corona. At 01:21 UT on 24 December the outward motion of large-scale loop-like coronal structures above the prominence began. The southern end of these large coronal loops appeared to be near 61S 47W. At around 01:21 UT on 24 December, the motion of prominence material near this footpoint began. These prominence activities continue through 03:13 UT on 24 December.

\section{Discussion}

These observations obtained with the EIT and LASCO experiments on SOHO provide a unique view of the earliest phases of a coronal mass ejection. To the best of our knowledge, similar observations have never been obtained in the past. The primary signature of CMEs in EUV and X-ray images is the creation of post-flare loop systems following the eruption of CMEs (Sheeley et al., 1975; Kahler, 1977; Webb, Krieger, and Rust, 1976; Tsuneta et al., 1992). More recently, transient voids of coronal X-ray emitting material are understood to signal the launch of a CME (Hudson, Lemen, and Webb, 1997).

Coronal mass ejections are clearly large-scale phenomena. At the Sun they are often related to streamers which they can completely disrupt and, once launched, can traverse the heliosphere at least to $1 \mathrm{AU}$. In the present case where the CME has been observed by EIT and LASCO from its earliest phases, the initiation begins in a very small volume. The initiation of a large-scale CME in an small-scale eruption is a key fact that must be included in our understanding of the creation of CMEs.

The EIT and LASCO observations show that from essentially the very beginning, the 3 main common components of a CME are present: the bright loop-like outer shell, the coronal void and the eruptive prominence. This would indicate that the basic physical state of the CME is completely determined at its initiation and that during its transit of the heliosphere, the development of the CME is just the evolution of this physical state. More observations of the initiations of CME, particularly with better time cadences, would be important for understanding this important phenomena.

\section{References}

Brueckner, G. E. et al.: 1995, Solar Phys. 162, 357.

Delaboudinière, J.-P. et al.: 1995, Solar Phys. 162, 291.

Feynman, J. and Martin, S. F.: 1995, J. Geophys. Res. 100, 3355.

Hudson, H., Lemen, J., and Webb, D.: 1997, in B. Bentley and J. Mariska (eds), 'A Signature of CME Onset in Soft X-Rays', Magnetic Reconnection in the Solar Atmosphere, ASP Conf. Ser. 111, p. 379. 
Hundhausen, A.: 1987, in V. J. Pizzo, T. Holzer, and D. G. Sime (eds), 'The Origin and Propagation of Coronal Mass Ejections', Proceedings of the Sixth International Solar Wind Conference, NCAR TN-306, p. 181.

Kahler, S.: 1977, Astrophys. J. 214, 891.

Kahler, S.: 1987, in V. J. Pizzo, T. Holzer, and D. G. Sime (eds), 'Observations of Coronal Mass Ejections Near the Sun', Proceedings of the Sixth International Solar Wind Conference, NCAR TN-306, p. 215.

Low, B. C. and Hundhausen, A. J.: 1987, J. Geophys. Res. 92, 2221.

Sheeley, N. R., Jr., Bohlin, J. D., Brueckner, G. E., Purcell, J. D., Scherrer, V. E., Tousey, R., Smith, J. B., Jr., Speich, D. M., Tandberg-Hanssen, E., Wilson, R. M., De Loach, A. C., Hoover, R. B., and McGuire, J. P.: 1975, Solar Phys. 45, 377.

Sime, D. G. and Hundhausen, A. J.: 1987, J. Geophys. Res. 92, 1049.

Simnett, G. M. et al.: 1997, Solar Phys. 175, 685 (this issue).

Tsuneta, S., Takahashi, T., Acton, L., Bruner, M. E., Harvey, K. L., and Ogawara, Y.: 1992, Publ. Astron. Soc. Japan 44, L211.

Wagner, W. J.: 1984, Ann. Rev. Astron. Astrophys. 22, 267.

Webb, D. F., Krieger, A. S., and Rust, D. M.: 1976, Solar Phys. 48, 159. 